\title{
Improvement of insulation properties of conventional brick products
}

\author{
Viktor BÁNHIDI ${ }^{1, a}$, László A. GÖMZE ${ }^{1, b}$ \\ ${ }^{1}$ Department of Ceramics and Silicate Engineering, \\ Faculty of Materials Science and Engineering, University of Miskolc, \\ Miskolc-Egyetemváros, H-3515 Hungary \\ afemvitya@uni-miskolc.hu, ${ }^{b}$ femgomze@uni-miskolc.hu
}

Keywords: brick clay, thermal conductivity, agricultural byproducts, wastes, mechanical properties

\begin{abstract}
The use of technologically byproduct agricultural wastes in various segments of the brick and tile industry is increasing continuously. The additives, mixed into the raw clay ignite during the firing process, adding extra thermal energy from inside the mixture decreasing the energy requirements of the manufacturing process. Added to this, through the combustion of the bio-wastes the porosity increases enhancing the thermal insulation properties of the final product. We have investigated some common, agricultural wastes to determine their effect on the thermal properties of bricks. In our experiments industry relevant amounts of additives (sawdust, rice-peel, seed-shell) were added to the basic clay composition. We have prepared mixtures with additive concentrations of $0,4,7$ percentage by weight. The preparations of the samples were (milling, drying and firing) following industrial standard procedures. Precise thermal conductivity data were gathered from all samples using a RAPID-K type static thermal conductivity measuring instrument. Our measurements show that by increasing the amount of the organic byproducts in the clay mixture it is possible to significantly decrease their thermal conductivity, leading to an improved insulation capability of commercial brick products. On the other hand, there was only a minor reduction in the mechanical strength found during previous works. The investigated agricultural byproducts were also ranked based on their effect on the product's thermal properties. It was found that the largest decrease to the thermal conductivity was caused by the sunflower seed-shell additive. Mixing $7 \%$ wt. seed shell to the clay, we can decrease the thermal conductivity of the fired product from $0,27 \mathrm{~W} / \mathrm{m} \cdot \mathrm{K}$ to $0,17 \mathrm{~W} / \mathrm{m} \cdot \mathrm{K}(36 \%)$. We have found that under the same conditions the sawdust caused the least improvement, only a decrease of $0,27 \mathrm{~W} / \mathrm{m} \cdot \mathrm{K}$ to $0,23 \mathrm{~W} / \mathrm{m} \cdot \mathrm{K}(16 \%)$ was measured.
\end{abstract}

\section{Introduction}

Using renewable agricultural byproducts and waste materials as performance enhancing additives in the brick industry is gaining more and more ground recently [1,2,3]. The additives, mixed in the brick clay are burning out during the firing process producing extra energy, and decreasing the total energy need of the industrial furnace. At the beginning, sawdust, wood chips and other wood based materials were used, but more recently polymers and also renewable agricultural waste materials, like rice-peel or seed-shell are also present as additions in the brick and tile industry. The ignition of the additives provides extra thermal energy from inside the brick product, and decreases the required energy by the furnace. Beside of saving energy, the combustion removes the additives, creating pores, further decreasing the thermal conductivity and unfortunately also decreases the mechanical strength of the final product. Since the project was tied to the industry it seemed necessary to describe the thermal conductivity and other parameters primarily as function of the additive concentration, since this is a technological parameter that can be monitored, and can be changed easily during manufacturing. Series of experiments were executed to measure the effect of the type and the concentration of the applied waste materials on the thermal conductivity and 
mechanical properties of the final brick product. Several mixtures were prepared using mined clay minerals and additives (sawdust, rice-peel, seed-shell) with concentrations of $0,4,7 \% \mathrm{wt}$. The process used to prepare the sample products for the measurements was following industrial procedures. The differences in the properties caused by the types of material used as additives were also evaluated.

\section{Sample preparation}

Two types of clays were used for raw materials, a yellow and gray from two different parts of the industrial partner's mine. We used a Bruker D8 X-ray diffractometer to analyze phase distribution of the clays. The compositions are shown in figures 1 and 2.

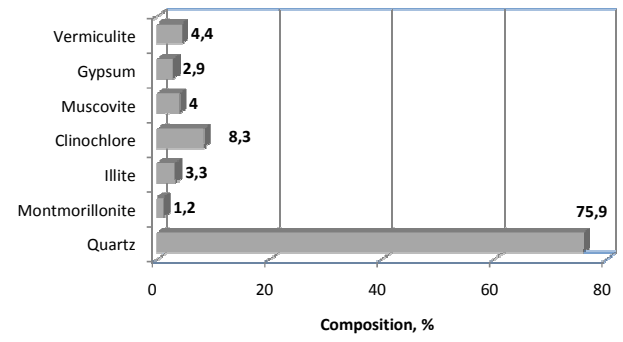

Figure 1.

Phase distribution of yellow clay

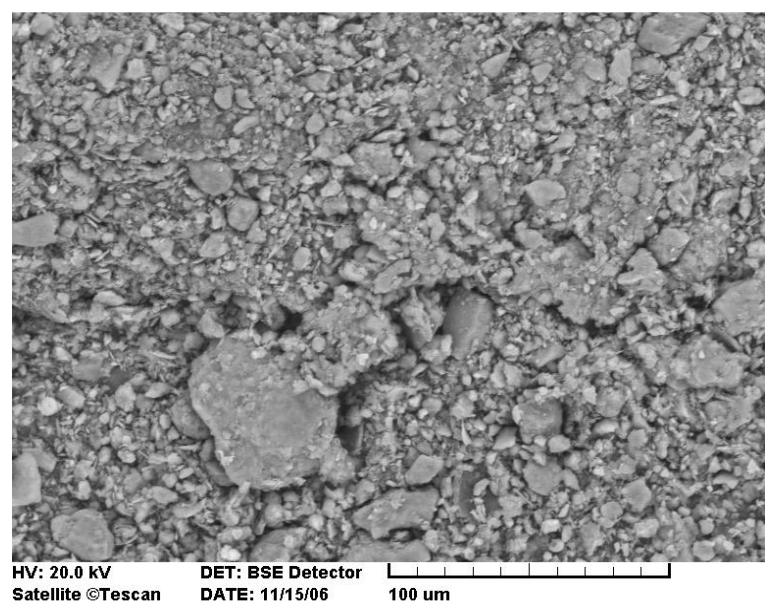

Figure 3.

Microstructure of yellow clay (1000x)

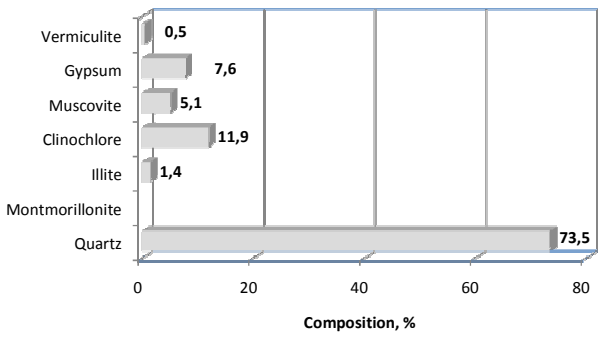

Figure 2.

Phase distribution of gray clay

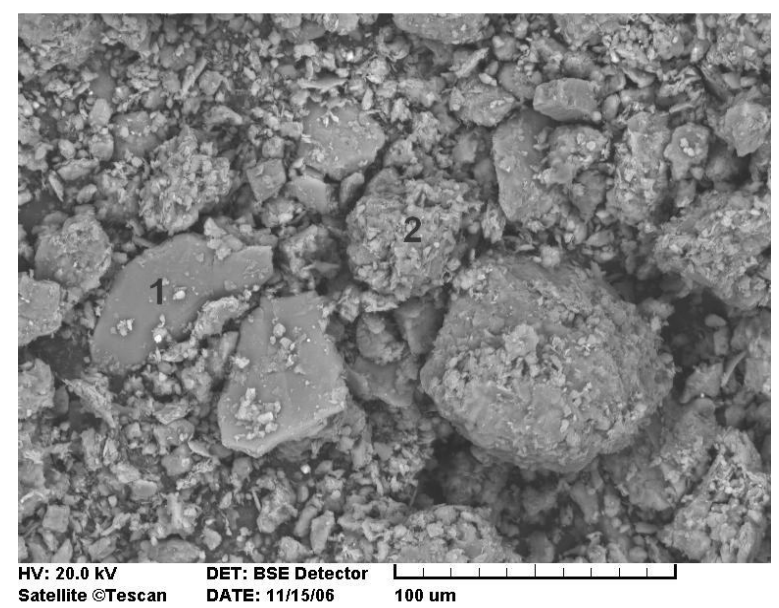

Figure 4.

Microstructure of gray clay $(1000 x)$

Milling in a pan mill was used to ensure the proper quality of the raw materials. To examine the microstructure of the used clays, Scanning Electron Microscopy (SEM) was used. The images of the microstructures taken are shown in figures 3 and 4.

It is easy to see on the SEM images, that homogeneous grain distribution wasn't achieved in the clays, as it was also not a requirement due to improve the packing factor of the powder system. In case of the yellow clay we found that the dominant grain size is below $20 \mu \mathrm{m}$, but there are also grains larger than $50 \mu \mathrm{m}$. The gray clay has larger inhomogenity, the average grain size is larger due to the dominance of the large particles $(>20 \mu \mathrm{m})$. The surface of some of the larger grains collects the smaller ones. See the indicated grains where \#2 is covered and \#1 is not that much. The pore forming additives also required some preparation. Sieving and drying was used to set the 
particle size distribution and the humidity of the additives. Three types of agricultural wastes were chosen for testing. These materials are all produced in abundance every year by the agricultural industry, and build up a large reservoir for industrial applications. Mixing them into prepared clay, these agricultural wastes can act as pore formers; thereby influencing the final properties of the brick product.

As next step the additives were mixed into the prepared factory clay mixtures (yellow and gray clay minerals in ratio of 4:1), creating a number of clay based recipes with additive concentrations of 0 , $4,7 \%$ wt. and with water contents between $15.57-16.67 \%$ wt. The mineral content of the used clay component is shown in figure 5.

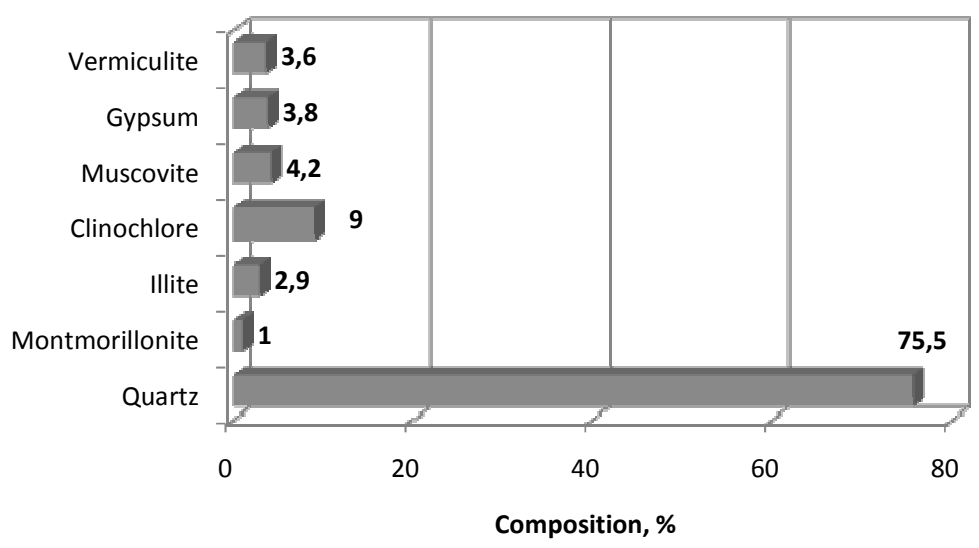

Figure 5.

Mineral content of the used clay component

Construction material testing standard sized specimens were produced for the thermal measurements, with dimensions of $300 \times 300 \times 50 \mathrm{~mm}$ using a special "molding" box. For the mechanical tests, $50 \mathrm{~mm}$ and $150 \mathrm{~mm}$ long rod shaped samples with a diameter of $33 \mathrm{~mm}$ were produced with common extrusion technology. All the samples were dried and fired using the same conditions. Table 1 lists the compositions of the samples.

Table 1. Compositions of the prepared samples

\begin{tabular}{|c|c|c|c|c|c|c|c|}
\hline $\begin{array}{c}\text { Pore forming } \\
\text { additives }\end{array}$ & \multicolumn{2}{|c|}{ Sawdust } & \multicolumn{2}{|c|}{ Rice-peel } & \multicolumn{2}{|c|}{ Seed-shell } & Reference \\
\hline $\begin{array}{c}\text { Clay } \\
\text { component }\end{array}$ & \multicolumn{5}{|c|}{ Mixture of yellow and gray clay minerals in ratio of 4:1 } \\
\hline $\begin{array}{c}\text { Additive } \\
\text { concentration }\end{array}$ & $4 \%$ & $7 \%$ & $4 \%$ & $7 \%$ & $4 \%$ & $7 \%$ & $0 \%$ \\
\hline Sample No. & F4 & F7 & R4 & R7 & N4 & N7 & 0 \\
\hline
\end{tabular}

The samples were first dried under laboratory conditions for 72 hours. To ensure a crack free surface this time was crucial to hold, and the process required great care. The second stage of drying was in a - humidity and temperature controlled - drying chamber at $90^{\circ} \mathrm{C}$ The samples were fired at a standard industrial temperature of $900^{\circ} \mathrm{C}$ with a $100^{\circ} \mathrm{C} / \mathrm{h}$ heating rate and using 2 hours soaking time. For the firing a laboratory muffle furnace (Hoker 2/3 1200) was used. Thanks to the careful drying, the fired samples were crack free, and compact with a smooth and plain surface. 


\section{Thermal conductivity measurements}

A low temperature RAPID-K type heat conductivity measurement system was used to determine the thermal conductivity of our samples (figure 6). The instrument was designed for static experiments, equipped with a measurement chamber, where the temperatures of the parallel and opposite sides were controlled by electric heating, and also water cooling, ensuring a homogenous temperature field (small fluctuations). The bottom plate contains a heat flux detector to collect quantitative data about the amount of heat transferred. The sample sits between the two temperature controlled plates (heat buffers) and a controlled heat exchange starts between the buffer plates through the brick sample due to a fix temperature gradient.

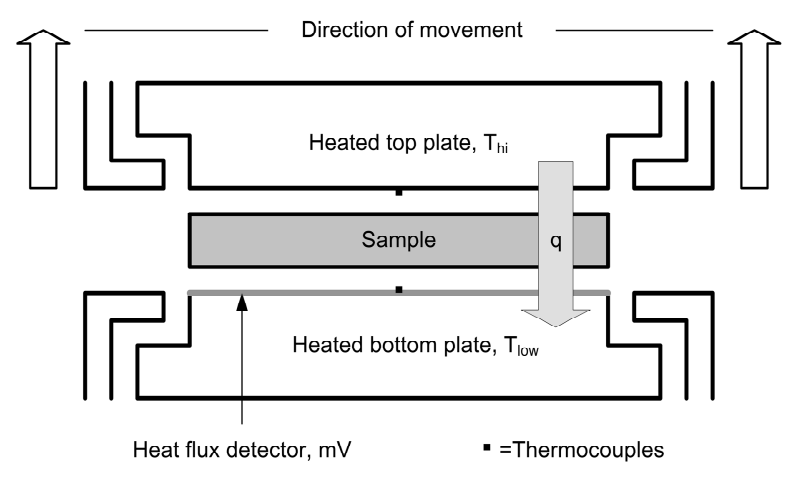

Figure 6.

Drawing of the RAPID-K device

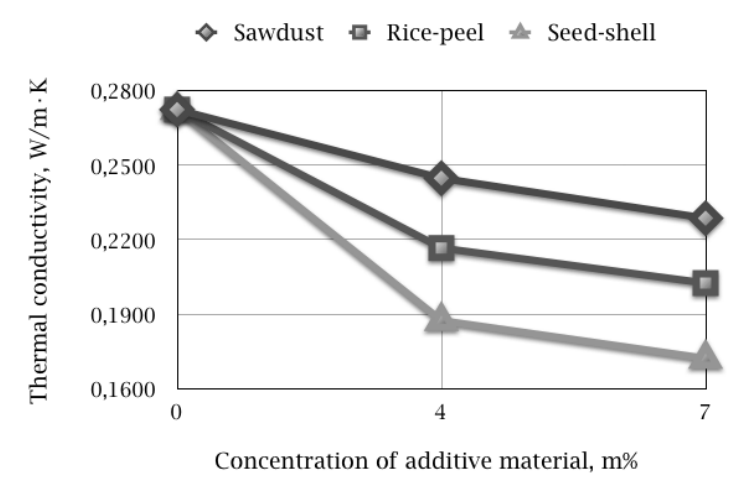

Figure 7.

Thermal conductivity of the measured products

The one dimensional heat transfer was ensured by effective isolation of the vertical side walls. As the walls have had the same thermal gradient and condition as the tested materials, the one dimensional solution of the thermal conductivity differential equations can be used. As the thermal resistance depends on the temperature, a calibrational step measuring of a known standard sample was necessary.

We used fast response $\mathrm{K}$ type thermocouples to measure the actual temperatures and the heat flux, the data was collected by a multi channel digital data acquisition system. After the steady state was reached (about $1 \mathrm{~h}$ ), the values of interest could be determined from the constant part of the temperatures and the voltage data of the heat flux detector plotted vs. time. Using equation 1 and the instrument specific conversion coefficient, the thermal resistivity of the "unknown" material can be determined.

$$
\mathrm{R}=\Delta \mathrm{T} /(\mathrm{U} \cdot \mathrm{k})
$$

where, $R$ is the thermal resistivity of the sample, $\Delta T$ is the temperature difference between the two opposite walls of the sample, $U$ is the measured heat flux and $k$ is the detector specific constant. Knowing the mean thickness of the sample, the thermal conductivity $(\lambda)$ is calculated using the following formula (eq. 2):

$$
\lambda=\Delta x / R
$$

where, $\lambda$ is the thermal conductivity, $\Delta x$ is the mean thickness and $R$ is the thermal resistivity of the sample. All measurements were carried out at the average temperature of $61^{\circ} \mathrm{C}$, and with a fixed $12^{\circ} \mathrm{C}$ temperature difference. This ensured the comparability of the results.

\section{Mechanical tests}

After firing the specimens were ready for mechanical measurements. The most important parameter for brick products as construction materials is the compressive strength. The measurements were executed in a combined tensile and compression tester with a maximum force of $100 \mathrm{kN}$ at the 
department's laboratory. Measuring the maximum force at the time of failure, and determining the average cross section of the sample the compressive strength could be calculated by eq. 3 .

$$
\sigma_{\text {comp }}=\frac{F}{A}
$$

where, $\sigma_{c o m p}$ is the compressive strength, and $A$ is the sample's initial cross section.

Since constructional materials as brick also has to resist bending forces too, bending measurements are also necessary. For measuring the flexural strength a three point bend setup was chosen. During the measurement we have registered the force, which was used to calculate the momentum caused by the load, and using a shape dependent value of the cross section factor the flexural strength can be determined as shown in equation 4 .

$$
\sigma_{\text {flex }}=\frac{0,25 \cdot F_{\text {flex }} \cdot l}{\frac{D^{3} \cdot \pi}{32}}
$$

where, $\sigma_{\text {flex }}$ is the flexural strength, $F$ is the measured force, $l$ is the support span, $D$ is the initial diameter of the sample and the $D^{3} \pi / 32$ is the cross section factor for cylindrical bodies.

\section{Results and discussion}

All measurements were performed for all material compositions using sawdust, rice-peel and seed-shell pore forming additives. The results of the heat conductivity measurements show; that the increase in the quantity of organic byproducts in the clay mixture significantly decreases the thermal conductivity. From the value of $0.27 \mathrm{~W} / \mathrm{m} \cdot \mathrm{K}$ of the unadulterated brick composition the value could be decreased by $10-31 \%$ with $4 \%$ wt. of additives. With the addition of $7 \%$ wt. of byproducts, the heat conductivity could be decreased by $16-37 \%$ from its original value. This indicates an improvement in the thermal properties compared to the industrially produced brick products. The thermal results are plotted in figure 7.

The pore forming additives were also ranked based on their heat insulation enhancing effect. Our measurements indicate that the seed-shell additive had affected the insulating properties the most (up to $37 \%$ less thermal conductivity). By the same additive concentration the rice-peel gave $26 \%$ decrease and the sawdust had an effect of only $16 \%$ decrease from the original value. The evaluation of the measured data listed above led us to the conclusion, that the sawdust additive was the least effective in decreasing the thermal conductivity.

The mechanical tests also show the same decrease of the measured properties as a function of concentration of the added organic waste materials. Unfortunately in these cases the decrease is a negative effect, as it leads to a lower quality product. Also we can see that as it was expected, the largest changes are caused by the same additive what improved the thermal insulation the most. The similarity of the change in properties is caused in all cases by the pore structure as it determines all these properties. In case of the seed shell additive there are relatively more pores (empty spaces) in the material, leading to a better insulating and worse compressive and flexural strengths. Since the compressive strength changes so radically (a decrease to the $77-26 \%$ of the original value for the $4 \%$ wt. and a decrease to the $48-25 \%$ of the original value for the $7 \% \mathrm{wt}$. of additives) with the increasing concentrations of rice peel and seed shell, further investigations are needed to determine the pore structures, and their real life applicability to modify brick products. Similar results were found in the flexural tests, with an interesting increase for the rice peel additive at $4 \%$ wt., which requires further studies. From the point of view of the mechanical properties the most applicable additive is the saw dust.

The differences of effects on the properties of the products might be explained by the differences in the agglomeration capabilities, in the density and in the water uptake and swelling of the three examined additives. 


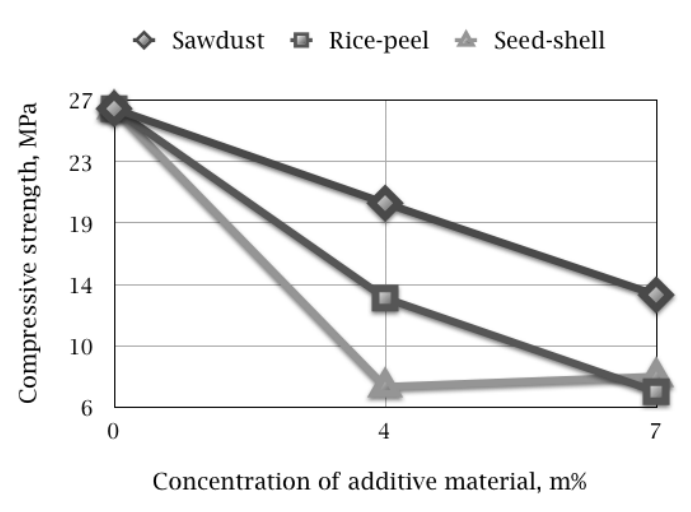

Figure 8.

Compressive strength of the measured brick products

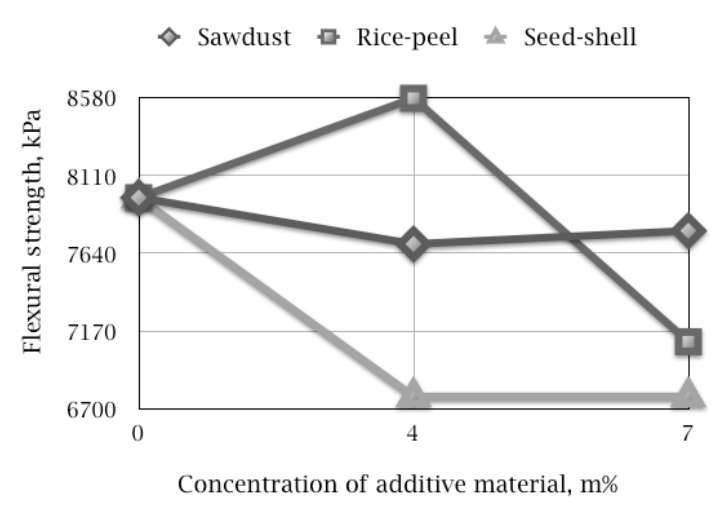

Figure 9.

Flexural strength of the examined brick compositions

These above mentioned properties should strongly define the pore volume and size distribution within the final product. Further studies are required to evaluate these relations.

\section{Conclusion}

Resulting in better thermal properties for the product, and providing extra heat during the firing process; the usage of renewable agricultural, industrial wastes as pore forming and/or reinforcing additives, in industrial scale could be advantageous, and a perspective area of continuing research in the brick industry $[1,3,4]$ even with their effect on the mechanical properties of the fired brick. Results show that the mixing of additives improves the insulation properties of the bricks. In this low concentration range (less than $10 \%$ wt.) these additives might decrease the mechanical properties below the levels required by standards, this issue needs to be addressed by further studies using testing samples of real brick product geometries. In case that the materials examined in this study are not applicable there are still more agricultural byproduct (corn leaf, corn stem, etc...) which still might prove advantageous to the brick industry.

\section{Acknowledgements}

The authors would like to thank to our industrial partner the Leier Hungária Kft. and to Szilárd Nagy for their generous support. The SEM measurements were performed in the laboratory of the Department of Physical Metallurgy and Metallforming by Á. Kovács. The XRD data was generously supplied by V. Mádai of the Department of Mineralogy and Petrology.

\section{References}

[1] Xu Lingling, Guo Wei, Wang Tao and Yang Nanru: Study on fired bricks with replacing clay by fly ash in high volume ratio, Construction and Building Materials, Volume 19, Issue 3, April 2005, pp. 243-247

[2] Ismail Demir, M. Serhat Baspınar and Mehmet Orhan: Utilization of kraft pulp production residues in clay brick production, Building and Environment, Volume 40, Issue 11, November 2005, pp.: 1533-1537

[3] Asokan Pappu, Mohini Saxena and Shyam R. Asolekar: Solid wastes generation in India and their recycling potential in building materials, Building and Environment, Volume 42, Issue 6, June 2007, pp.: 2311-2320

[4] Hanifi Binici, Orhan Aksogan, Mehmet Nuri Bodur, Erhan Akca and Selim Kapur: Thermal isolation and mechanical properties of fibre reinforced mud bricks as wall materials, Construction and Building Materials, Volume 21, Issue 4, April 2007, pp. 901-906 